In Cres. Vol. $4 N^{\circ}$ 2: pp. 269-283, 2013

\title{
PERFIL DE LA PLANIFICACIÓN Y ORGANIZACIÓN DE LAS TECNOLOGÍAS DE INFORMACIÓN Y COMUNICACIÓN EN LA UNIVERSIDAD*
}

\author{
PROFILE OF PLANNING AND ORGANIZATION OF INFORMATION \\ AND COMMUNICATION TECHNOLOGY AT UNIVERSITY
}

Jorge Luis Gutiérrez Gutiérrez ${ }^{l}$

\section{RESUMEN}

La presente investigación determina el perfil de la planificación y de la organización de las tecnologías de la información y comunicaciones en la Universidad Católica Los Ángeles de Chimbote, tomando como referencia un instrumento de recolección de datos validado y que delimita la medición al perfil de la gestión de la planificación y de la organización de acuerdo al marco de referencia Cobit. Esta investigación pertenece a la línea de investigación en tecnologías de información y comunicación (TIC) de la Escuela Profesional de Ingeniería de Sistemas de la Universidad Católica los Ángeles de Chimbote.

El estudio es de tipo cuantitativo, descriptivo, no experimental, de corte transversal y en él se analiza el nivel de perfil de acuerdo a cinco criterios: definición de plan estratégico de TI, arquitectura de la información, dirección tecnológica, procesos, organización y relaciones de TI, evaluación y administración de riesgos de TI.

Para la medición de las variables de estudio se utilizaron encuestas, las cuales fueron remitidas a través de documentos físicos al Jefe de la División de Sistemas y, por medio de él, al personal a su cargo de todas los departamentos que conforman esta división. Se aplicó una sola encuesta directa y escrita guiada por el encuestador.

* Recibido: 18 de mayo del 2013; aprobado: 03 de octubre del 2013.

1 Doctor en Ingeniería. Universidad Nacional Federico Villarreal. Docente asociado de la escuela de Ingeniería de Sistemas de la Universidad Católica Los Ángeles de Chimbote. 
Se trabajó con una muestra constituida por 11 trabajadores, seleccionados tomando en cuenta su involucramiento con los procesos de TI de la universidad.

Los resultados han demostrado que la ULADECH Católica presenta las variables en estudio en un nivel definido; se afirma que la universidad está en un estadío auspicioso donde se fijan objetivos claros, así como estrategias idóneas para alcanzarlos.

Se está consolidando la arquitectura empresarial de TI que permitirá alinear los sistemas de información y comunicación a los procesos primarios y de soporte de la universidad; además se tienen alineados los objetivos de la división de sistemas a los de la universidad; se están determinando los riesgos de TI.

PALABRAS ClAVE: Tecnologías de información y comunicaciones, Plan estratégico de TI, Arquitectura de la información, Dirección tecnológica, Procesos, organización y relaciones de TI.

\section{ABSTRACT}

This study belongs to line of research in information and communication technologies (ICT) of the of Systems Engineering at Catholic University of Angels from Chimbote Perú ULADECH Católica-, it aims to describe the profile of planning and organization of ICT at system division at ULADECH Católica.

This research has focused qualitatively, descriptive, not experimental, cross sectional and it analyzes the level of profile of these five variables: definition of IT strategic plan, information architecture, technology direction, processes, organization and relationships IT, assess and manage IT risks.

To measure and control variables of this study, was used survey, which was transmitted through by physical documents to CIO Donny Acosta Benites and his responsable and direct managers and management issues ICT at ULADECH Católica; it was applied a single validated test directly guided by the interviewer.

We worked with a sample of 11 employees of this university, selected based on their involvement with the IT processes.

The results had demonstrated that ULADECH Católica has the 5 variables of study in definited level; we concluded that the university is an auspicious stage that sets clear objectives and appropriate strategies to achieve them.

It is consolidating IT enterprise architecture that will align the information and communication systems to primary and support processes of the university; it has also aligned the objectives of the systems division of the university are being identified risks IT.

KEY WORDS: Information and communications technologies, IT strategic plan, information architecture, technology direction, processes, IT organization and relationships. 


\section{INTRODUCCIÓN}

En el Perú hay 133 universidades según la última estadística de la Asamblea Nacional de Rectores al año 2011, entre públicas y privadas; todas con buena cantidad de procesos y de datos comunes como, por ejemplo, el control de pagos del estudiante, el proceso de matrícula, el control del kardex de notas, el control de la admisión, el control de grados y títulos, así como muchos otros procesos enmarcados básicamente en el modelo de calidad del CONEAU y similares.

Según Márquez ${ }^{1}$, refiriéndose a la tecnología: "Sus principales aportaciones a las actividades humanas se concretan en una serie de funciones que nos facilitan la realización de nuestros trabajos, porque sean estos los que sean, siempre requieren una cierta información para realizarlo, un determinado proceso de datos y a menudo también la comunicación con otras personas; y esto es precisamente lo que nos ofrecen las TIC".

Posteriormente, con el advenimiento de Internet, el proceso se realiza en servidores distribuidos por todo el mundo; si bien es cierto que se han evidenciado muchos beneficios del uso de las tecnologías, también se ha suscitado una serie de problemas que ponen en riesgo la información y como consecuencia, la prestación de servicios.

La página de Ibermática ${ }^{2}$, tratando el tema de oportunidades y amenazas sociales de la TIC, concluye: "El desarrollo que aportan la TIC transforma poderosamente los modos de vida y la actividad laboral y profesional. Sin embargo, no hay una suficiente conciencia práctica a la hora de abordar esos cambios, ni en la dirección a emprender ni en la urgencia de los mismos".

La problemática de las tecnologías es mundial y genera nuevas formas de dependencia. Según un estudio ${ }^{3}$ de la UIT (Unión Internacional de Telecomunicaciones), la tecnología crece en un $30 \%$ manteniendo una brecha digital paralela entre los países desarrollados y los menos favorecidos. Para minimizar este problema se ha implementado un Plan Marshall ${ }^{4}$ de la tecnología para África.

Salazar ${ }^{5}$ comenta que "las TIC agregan valor a las actividades operacionales y de gestión empresarial en general y permite a las empresas obtener ventajas competitivas, permanecer en el mercado y centrarse en su negocio".

En este sentido, la información es un gran eje sobre el que giran muchas actividades del quehacer humano. Desde la aparición de las computadoras se inició una revolución en el tratamiento y uso de la información en las organizaciones. La experiencia de las empresas respecto al uso de las tecnologías ha sido 
muy variada en cuanto a la satisfacción del servicio ${ }^{6}$; sin embargo, es una necesidad de la que no se puede prescindir; prácticamente no hay empresas que no utilicen computadoras.

Para las universidades es vital la existencia de una gran comunicación interna entre sus departamentos y externa con sus clientes y proveedores; además, es importante que el flujo de información cada vez sea más rápido para que se puedan identificar los problemas en el menor tiempo posible y se puedan tomar decisiones oportunas para resolverlos, de manera que es necesario invertir en las TIC, generando más beneficio para la empresa, mayores ingresos, procesos de negociación a corto plazo, comunicación rápida con los proveedores, confiable y con mayor seguridad, mejores servicios al cliente.

Actualmente existe una gran cantidad de dinero invertido en TI alrededor del mundo ${ }^{7}$. Las empresas siguen considerando que es una inversión altamente provechosa, aunque en la mayoría de las veces sólo en el largo plazo. En algunos países es el área con mayor capital invertido en los negocios, puesto que se necesita de una inversión significativa para poder desarrollarla y obtener los soportes requeridos (el hardware disponible, infraestructura de telecomunicaciones, arquitectura de sistemas, etc.), para llegar a ser una herramienta competitiva, obteniendo información sobre el control e integración empresarial.

La planeación y organización de TIC es la fuente principal para lograr el objetivo de los negocios. COBIT está diseñado para ser utilizado no sólo por proveedores de servicios, usuarios y auditores de TI, sino también, y principalmente, como guía integral para la gerencia y para los dueños de los procesos de negocio. Para proporcionar la información que la empresa requiere para lograr sus objetivos, la empresa necesita invertir en TIC y administrar y controlar los recursos de TI usando un conjunto estructurado de procesos que provean los servicios que entregan la información empresarial requerida. El marco de trabajo COBIT $^{8}$ ofrece herramientas para garantizar la alineación con los requerimientos del negocio.

En este contexto, la ULADECH Católica viene desarrollando una interesante arquitectura empresarial de tecnologías de la información, que responde a un proceso evolutivo pasando desde la masificación del uso de internet, hasta el uso compartido del ERP University, un sistema de información para muchos usuarios con diferente perfil que alcanza inclusive a los estudiantes.

Es importante verificar ahora, en el 2012, cómo se encuentra el nivel de planeamiento y organización, especificado en los siguientes cinco variables: 
Plan estratégico; arquitectura de la información; dirección tecnológica; procesos, organización y relaciones de TI; evaluación y administración del riesgo de TI.

El plan estratégico de TI es necesario para gestionar y dirigir todos los recursos de tecnología de información en línea con la estrategia y prioridades del negocio. En la actualidad muchas organizaciones no planifican la estrategia de TI como se debería, lo cual es importante para lograr los objetivos del negocio, generar mayor ingreso, costo bajo, mejor servicio al cliente en la organización.

La arquitectura de la información es importante para determinar la estructura coherente de módulos que soportan la tecnología de información en la organización.

En cuanto a la determinación de la dirección tecnológica, la división de sistemas o su equivalente debe estar consciente de la importancia del plan de infraestructura tecnológica. El proceso para el plan de infraestructura tecnológica es razonablemente sólido y está alineado con el plan estratégico de TI.

La orientación de la infraestructura tecnológica incluye el entendimiento dónde la empresa desea ser líder y dónde desea rezagarse respecto al uso de tecnología, con base en los riesgos y en la alineación con la estrategia organizacional.

Los procesos, organización y relaciones de TI en la organización deben tomar en cuenta los requerimientos del personal; debe existir la transparencia como el involucramiento de alto ejecutivo, gerencia del negocio, aseguramiento de calidad, administración de riesgo, seguridad de la información y garantía de los requerimientos del negocio.

En cuanto a evaluar y administrar los riesgos de TI, la organización debería contar con un marco de trabajo de administración de los riesgos, debiendo ser expresado en términos financieros para que pueda rendir cuenta concreta.

\section{PROBLEMA}

¿Cuál fue el perfil de la planificación y organización de las tecnologías de información y comunicación (TIC) en la Universidad Católica Los Ángeles de Chimbote en el año 2012?

En el siguiente cuadro se muestra la definición y la operacionalización de la variable de estudio. 
Jorge Luis Gutiérrez Gutiérrez

\begin{tabular}{|c|c|c|c|c|c|}
\hline VARIABLE & $\begin{array}{l}\text { DEFINICIÓN } \\
\text { CONCEPTUAL }\end{array}$ & DIMENSIONES & INDICADORES & $\begin{array}{c}\text { ESCALA } \\
\text { DE } \\
\text { MEDICIÓN }\end{array}$ & $\begin{array}{l}\text { DEFINICIÓN } \\
\text { OPERACIONAL }\end{array}$ \\
\hline \multirow[t]{5}{*}{$\begin{array}{l}\text { Planificación } \\
\text { y } \\
\text { organización } \\
\text { de las TI. }\end{array}$} & \multirow[t]{5}{*}{$\begin{array}{l}\text { Es el } \\
\text { conjunto de } \\
\text { estrategias y } \\
\text { tácticas, y la } \\
\text { manera en } \\
\text { que TI } \\
\text { contribuye } \\
\text { al logro de } \\
\text { los } \\
\text { objetivos } \\
\text { del negocio. }\end{array}$} & $\begin{array}{l}\text { Plan } \\
\text { estratégico } \\
\text { de TI. }\end{array}$ & $\begin{array}{l}\text { - Elabora plan estratégico de TI. } \\
\text { - Elabora plan táctico de TI. } \\
\text { - Elabora portafolios de proyectos } \\
\text { de TI. } \\
\text { - Elabora portafolios de servicios } \\
\text { de TI. } \\
\text { - Define estrategia de contratación } \\
\text { externa de TI. } \\
\text { - Define estrategia de adquisición } \\
\text { de TI. }\end{array}$ & \multirow{4}{*}{ Ordinal } & $\begin{array}{l}\text { Inexistente. } \\
\text { Inicial. } \\
\text { Intuitivo. } \\
\text { Definido. } \\
\text { Administrado. } \\
\text { Optimizado. }\end{array}$ \\
\hline & & $\begin{array}{l}\text { Arquitectura } \\
\text { de la } \\
\text { información }\end{array}$ & $\begin{array}{l}\text { - Tiene esquema de clasificación de } \\
\text { datos. } \\
\text { - Elabora plan de sistemas del } \\
\text { negocio optimizado. } \\
\text { - Define diccionario de datos. } \\
\text { - Define arquitectura de la } \\
\text { información. } \\
\text { - Asigna clasificación de datos. } \\
\text { - Define procedimientos y } \\
\text { herramientas de clasificación. }\end{array}$ & & $\begin{array}{l}\text { Inexistente. } \\
\text { Inicial. } \\
\text { Intuitivo. } \\
\text { Definido. } \\
\text { Administrado. } \\
\text { Optimizado. }\end{array}$ \\
\hline & & $\begin{array}{l}\text { Dirección } \\
\text { tecnológica }\end{array}$ & $\begin{array}{l}\text { - Utiliza estándares tecnológicos. } \\
\text { - } \text { Realiza actualizaciones del } \\
\text { estado de la tecnología. } \\
\text { - Tiene plan de infraestructura } \\
\text { tecnológica. } \\
\text { - Define requerimientos de } \\
\text { Infraestructura. }\end{array}$ & & $\begin{array}{l}\text { Inexistente. } \\
\text { Inicial. } \\
\text { Intuitivo. } \\
\text { Definido. } \\
\text { Administrado. } \\
\text { Optimizado. }\end{array}$ \\
\hline & & $\begin{array}{l}\text { Procesos, } \\
\text { organización } \\
\text { y } \\
\text { relaciones } \\
\text { de TI. }\end{array}$ & $\begin{array}{l}\text { - Define marco de trabajo. } \\
\text { - } \text { Asigna dueños de sistemas } \\
\text { documentados. } \\
\text { - } \text { Reglamenta la organización y } \\
\text { relaciones. } \\
\text { - Define marco de procesos, roles } \\
\text { y responsabilidades } \\
\text { documentados. }\end{array}$ & & $\begin{array}{l}\text { Inexistente. } \\
\text { Inicial. } \\
\text { Intuitivo. } \\
\text { Definido. } \\
\text { Administrado. } \\
\text { Optimizado. }\end{array}$ \\
\hline & & Riesgos de TI & $\begin{array}{l}\text { - Realiza evaluación de riesgos. } \\
\text { - Genera reportes de riesgos. } \\
\text { - Formula directrices de } \\
\text { administración de riesgos. } \\
\text { - Formula planes de acciones } \\
\text { correctivas para riesgos. }\end{array}$ & & $\begin{array}{l}\text { Inexistente. } \\
\text { Inicial. } \\
\text { Intuitivo. } \\
\text { Definido. } \\
\text { Administrado. } \\
\text { Optimizado. }\end{array}$ \\
\hline
\end{tabular}




\section{HIPÓTESIS}

El nivel del perfil de la planificación y la organización de las TIC se encuentra en el nivel medio, evidenciado por el mismo nivel de sus cinco procesos delimitados en el alcance de este estudio.

\section{OBJETIVO}

Determinar el nivel del perfil de la planificación y la organización de las tecnologías de la información y las comunicaciones en la Universidad Católica Los Ángeles de Chimbote en el año 2012.

\section{MATERIAL Y MÉTODO}

\section{TIPO DE INVESTIGACIÓN}

En esta investigación se aplica el método científico inductivo, puesto que se estudian características de los procesos referidos a la planeación y organización que pueden ser inferidos sobre los demás procesos que componen la primera dimensión del modelo Cobit. Se afirma que con resultados parciales en la muestra de estudio se generaliza a toda la población.

\section{DISEÑO DE INVESTIGACIÓN}

Investigación de tipo cuantitativo, descriptivo, no experimental, de corte transversal.

El diseño de investigación utilizado es el descriptivo de una sola casilla, el cual se grafica de la siguiente manera:

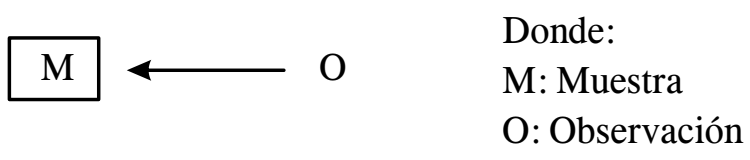

\section{POBLACIÓN Y MUESTRA}

Estuvo constituida por un total de 30 trabajadores de la división de sistemas de la Universidad Católica Los Ángeles de Chimbote.

Se utilizó el muestreo no probabilístico, por cuotas, porque se requirió una cuidadosa y controlada elección de los sujetos con las características especificadas en el planteamiento del problema.

Se determinó una muestra de 11 trabajadores, utilizando como criterio de inclusión el estar involucrado con los procesos de TI estudiados. 


\section{TÉCNICAS E INSTRUMENTOS UTILIZADOS}

Se utilizó la técnica de la encuesta, aplicada a cada uno de los empleados que constituyeron la muestra. Los instrumentos fueron aplicados a los trabajadores que conforman la muestra.

Se utilizó un listado de preguntas referentes a la definición de plan estratégico de TI, para determinar el perfil de dicho proceso. Este listado constó de 12 preguntas, con 6 posibles respuestas para cada una de ellas, que tienen un valor de 0 a $5(a=0, b=1, c=2, d=3, e=4, f=5)$.

\section{PROCESAMIENTO DE RECOLECCIÓN Y ANÁLISIS DE DATOS}

Se realizó una comunicación oficial al jefe de la División de Sistemas -ahora División de Sistemas de Información y Comunicación-, en la cual se le daba a conocer los alcances de esta investigación; luego, gracias a que el jefe nos facilitó un personal de confianza como apoyo para aplicar las encuestas al personal seleccionado de la división de sistemas, se procedió a dar un alcance para el correcto llenado dándoles a conocer la finalidad del estudio, así como los beneficios que lograría con los resultados del mismo.

Se convino una fecha y hora específica para visitas a locales a efectos de recolectar los datos para luego realizar un análisis y comprobar el nivel de definición de planeación y organización de TIC, en cada uno de los trabajadores, indicándoles que era parte de un proyecto de investigación.

\section{RESULTADOS}

A. VARIABLE DEFINICIÓN DE PLAN ESTRATÉGICO DE TI

Tabla 01

FRECUENCIA DEL PERFIL: DEFINICIÓN DE PLAN ESTRATÉGICO DE TI EN LA UNIVERSIDAD CATÓLICA LOS ÁNGELES DE CHIMBOTE EN EL AÑO 2012

\begin{tabular}{lcc}
\hline Nivel & Frecuencia & Porcentaje \\
\hline Inexistencia & 0 & 0,00 \\
Inicial & 0 & 0,00 \\
Repetible & 2 & 18,18 \\
Definido & 6 & 54,54 \\
Administrado & 3 & 27,27 \\
Optimizado & 0 & 0,00 \\
\hline TOTAL & 11 & 100,00 \\
\hline
\end{tabular}

Fuente: Cuestionario aplicado por el autor a la muestra seleccionada. 
En la tabla 01 se observa que el $18,18 \%$ del personal encuestado considera que el proceso de definición del plan estratégico de TI se encuentra en un nivel repetible, mientras que el 54,54\% se encuentra en un nivel definido y el $27,27 \%$ considera que se encuentra en un nivel administrado.

\section{B. VARIABLE DE ARQUITECTURA DE LA INFORMACIÓN}

Tabla 02

FRECUENCIA DEL PERFIL DE DEFINICIÓN DE LA ARQUITECTURA

DE LA INFORMACIÓN EN LA UNIVERSIDAD CATÓLICA

LOS ÁNGELES DE CHIMBOTE EN EL AÑO 2012

\begin{tabular}{lcc}
\hline Nivel & Frecuencia & Porcentaje \\
\hline Inexistencia & 0 & 0,00 \\
Inicial & 2 & 18,18 \\
Repetible & 1 & 9,09 \\
Definido & 4 & 36,36 \\
Administrado & 4 & 36,36 \\
Optimizado & 0 & 0,00 \\
\hline TOTAL & 11 & 100,00 \\
\hline
\end{tabular}

Fuente: Cuestionario aplicado a la muestra seleccionada. Aplicado por Jorge L. Gutiérrez Gutiérrez.

En la tabla 02 se observa que el $18,18 \%$ del personal encuestado considera que el proceso de definición de la arquitectura de la información se encuentra en un nivel inicial, mientras que el 9,09\% se encuentra en un nivel repetido; el 36,36\% considera que el proceso está en un nivel definido, y con el mismo porcentaje se considera que se está en un nivel administrado.

\section{VARIABLE DE DiRECCIÓN TECNOLÓGICA}

En la tabla 03 se observa que el $27,27 \%$ del personal encuestado considera que el proceso de definición de la dirección tecnológica se encuentra en un nivel repetible, mientras que el $36,36 \%$ se encuentra en un nivel definido; igual porcentaje manifiesta que se encuentra en un nivel administrado. 
Tabla 03

FRECUENCIA DEL PERFIL DE LA DIRECCIÓN TECNOLÓGICA EN LA UNIVERSIDAD CATÓLICA LOS ÁNGELES DE CHIMBOTE EN EL AÑO 2012

\begin{tabular}{lcc}
\hline Nivel & Frecuencia & Porcentaje \\
\hline Inexistencia & 0 & 0,00 \\
Inicial & 0 & 0,00 \\
Repetible & 3 & 27,27 \\
Definido & 4 & 36,36 \\
Administrado & 4 & 36,36 \\
Optimizado & 0 & 0,00 \\
\hline TOTAL & 11 & 100,00 \\
\hline Fuente: Cuestionario aplicado a la muestra seleccionada. Aplicado por Jorge L. \\
Gutiérrez Gutiérrez.
\end{tabular}

\section{VARIABLE PROCESOS, ORGANIZACIÓN, RELACIONES TI}

En la tabla 04 se observa que el $27,27 \%$ del personal encuestado considera que el proceso de definición de procesos, organización y relaciones de TI se encuentra en un nivel repetible, mientras que el $45,45 \%$ se encuentra en un nivel definido, el 18,18\% considera que el proceso está en un nivel administrado y el 9,09\%, que se está en un nivel optimizado.

Tabla 04

FRECUENCIA DEL PERFIL DE PROCESOS, ORGANIZACIÓN Y RELACIONES DE TI EN LA UNIVERSIDAD CATÓLICA LOS ÁNGELES DE CHIMBOTE EN EL AÑO 2012

\begin{tabular}{lcc}
\hline Nivel & Frecuencia & Porcentaje \\
\hline Inexistencia & 0 & 0,00 \\
Inicial & 0 & 0,00 \\
Repetible & 3 & 27,27 \\
Definido & 5 & 45,45 \\
Administrado & 2 & 18,18 \\
Optimizado & 1 & 9,09 \\
\hline TOTAL & 11 & 100,00 \\
\hline Fuente: Cuestionario aplicado a la muestra seleccionada. Aplicado por Jorge L. \\
Gutiérrez Gutiérrez.
\end{tabular}




\section{E. VARIABLE EVALUAR Y ADMINISTRAR LOS RIESGOS DE TI}

En la tabla 05 se observa que el 9,09\% del personal encuestado considera que el proceso de definición de evaluar y administrar los riesgos de TI se encuentra en un nivel inexistente, mientras que el $27,27 \%$ se encuentra en un nivel repetible, el $18,18 \%$ considera que el proceso está en un nivel definido y el $45,45 \%$, que el proceso está en un nivel administrado.

Tabla 05

FRECUENCIA DEL PERFIL DE EVALUAR Y ADMINISTRAR LOS RIESGOS DE TI EN LA UNIVERSIDAD CATÓLICA LOS ÁNGELES DE CHIMBOTE EN EL AÑO 2012

\begin{tabular}{lcc}
\hline Nivel & Frecuencia & Porcentaje \\
\hline Inexistencia & 1 & 9,09 \\
Inicial & 0 & 0,00 \\
Repetible & 3 & 27,27 \\
Definido & 2 & 18,18 \\
Administrado & 5 & 45,45 \\
Optimizado & 0 & 0,00 \\
\hline TOTAL & 11 & 100,00 \\
\hline Fuente: Cuestionario aplicado a la muestra seleccionada. Aplicado por Jorge L. \\
Gutiérrez Gutiérrez.
\end{tabular}

\section{DISCUSIÓN}

Este estudio se enfocó a determinar el perfil de la planificación y la organización de las tecnologías de la información y las comunicaciones en la ULADECH Católica. Para tal fin se ha recolectado información de cinco procesos correspondientes a dicho perfil como son: la definición del plan estratégico de TI, la arquitectura de la información, la dirección tecnológica, los procesos, organización y relaciones de TI, y la evaluación y administración de riesgos de TI en la Universidad Católica Los Ángeles de Chimbote.

Los resultados obtenidos en el presente estudio muestran que el 18,18\% del personal de la universidad considera que el proceso de planeación estratégica de TI se encuentra en un nivel repetible, mientras que el 54,54\% se encuentra en un nivel definido y un $27,27 \%$, un nivel administrado (tabla 01); se ha desarrollado la planeación estratégica de TI necesaria para gestionar y dirigir todos los recursos de TI en línea con la estrategia y prioridades del negocio. Estos 
resultados distan de los obtenidos en el estudio realizado en la empresa agrícola y ganadera "Chavín de Huántar" en el Año 2011, que señalan que el nivel de gestión de las TIC está en un grado de madurez inicial. Esta diferencia es comprensible, ya que la universidad ha apostado por el uso intensivo de TIC en todos sus procesos, desarrollando a la fecha una arquitectura empresarial como base para integración de procesos y servicios.

Asimismo, el $18.18 \%$ del personal de la universidad considera que la arquitectura de la información está en un nivel inicial, mientras que el 9,09\% considera que se encuentra en un nivel repetible; el 36,36\% considera que se encuentra en un nivel definido y el mismo porcentaje que se encuentra en un nivel administrado (tabla 02). Esto no coincide con los resultados del estudio realizado ${ }^{9}$ en la empresa ACUAPESCA SAC de la provincia de Casma en el año 2010, el cual tuvo como resultados que el $54 \%$ del personal de la empresa considera que el proceso de arquitectura de la información se encuentra en el nivel definido, pues el giro de la empresa elegida para el estudio es completamente distinto.

De igual modo, el 27,27\% del personal de la universidad considera que el proceso dirección tecnológica está en un nivel repetible, mientras que el $36,36 \%$ que se encuentra en un nivel definido y el mismo porcentaje, que se encuentra en un nivel administrado (tabla 03). Esto no coincide con los resultados obtenidos en algunas MYPES, como Shopping Center, toda vez que la realidad en las MYPES es mucho menos prodigiosa en cuanto al uso de TIC.

De igual modo, el 27,27\% del personal de la universidad considera que los procesos, organización y relaciones de TI están en un nivel repetible, mientras que el $45,45 \%$, que se encuentra en un nivel definido; el 18,18\% considera que se encuentra en un nivel administrado y un $9,09 \%$ que se encuentra en un nivel optimizado (tabla 04). Estos resultados no coinciden con los obtenidos en el estudio realizado en el Hospital La Caleta de Chimbote - $2011^{10}$, donde el $69,57 \%$ del personal administrativo considera que la variable procesos, organización, relaciones TI se encuentra en un nivel 3 (Definido), pero carece de conciencia al ejecutar el respectivo control y/o monitoreo.

Según la tabla 05, el 9,09\% de los empleados encuestados de la universidad considera que el proceso de definición de evaluar y administrar los riesgos de TI se encuentra en un nivel inexistente; además, el 27,27\% considera que se está en un estado repetido, mientras que el $18,18 \%$ se encuentra en un nivel definido y el 45,45\% considera que está en un nivel administrado. 
Estos resultados distan algo respecto de los obtenidos en las municipalidades de Santa $^{11}$ y Coishco $^{12}$, en las cuales, por ser organizaciones muy pequeñas, que no tienen como factor crítico a las TIC, sus procesos son todavía iniciales.

La ULADECH Católica alcanza, en promedio, que la planificación y la organización de las tecnologías de la información y comunicaciones de este estudio están en el nivel definido en lo que corresponde al año 2012. Estos resultados se justifican, puesto que la universidad, en cuanto al área que gestiona las TIC, es bastante grande contando con más de 30 empleados, habiendo logrado la integración de su sistema de información administrativo y académico -ERP University-, la integración de las redes informáticas de los 4 locales con los que cuenta en la ciudad de Chimbote, facilitando la comunicación y el internet a más de 500 computadoras, apuntando hoy a desarrollar una ya conceptualizada arquitectura empresarial de TI.

¿Qué se puede hacer para potenciar estos resultados? Luego de ser entrevistado el jefe de la división de sistemas, manifestó que entre otras actividades debería consistenciarse y socializarse entre todos los miembros de la división los planes estratégicos y operativos de esta división; además se debe elaborar los documentos normativos y de procesos; por ejemplo, definir las políticas de uso de TIC en la ULADECH Católica, además de elaborar el mapa de procesos de la universidad para alinearlos a la TI en un modelo de arquitectura empresarial de TI; por último, identificar y socializar los riesgos de TI en base a las prioridades del corporativo para poder garantizar la continuidad de las operaciones ante situaciones contingentes que conlleven la interrupción brusca de los servicios de TI a todos sus usuarios.

\section{CONCLUSIONES}

1. El $18,18 \%$ del personal encuestado considera que el proceso de definición del plan estratégico de TI se encuentra en un nivel repetible, mientras que el $54,54 \%$ se encuentra en un nivel definido y el $27,27 \%$ se encuentra en un nivel administrado del modelo COBIT; es decir, existe evidencia que la universidad ha definido claramente los objetivos de TI alineándolos a los de la organización. Estos resultados concuerdan con la hipótesis formulada sobre el nivel de este proceso, por lo que ésta se acepta.

Falta asegurar el análisis de riesgos de TI, no especificando las evaluaciones de riesgo en cada proyecto nuevo. La administración de TI especifica la res- 
ponsabilidad por la administración del riesgo evidenciándose en el MOFGC de la División de Sistemas.

2. El 36,36\% del personal encuestado considera que el proceso de definición de la arquitectura de la información se encuentra en un nivel definido y el mismo porcentaje considera que se está en un nivel administrado, mientras que el $18,18 \%$ se encuentra en un nivel inicial. Estos resultados no concuerdan con la hipótesis formulada sobre el nivel de este proceso, habiéndose obtenido resultados por encima de lo esperado, por lo que ésta se acepta según COBIT; es decir, la organización tiene definida su arquitectura empresarial de TI.

3. El 36,36\% del personal encuestado considera que el proceso de definición de la dirección tecnológica se encuentra en un nivel definido; igual porcentaje manifiesta estar en el nivel administrado, mientras que el 27,27\% manifiesta que se encuentra en un nivel inicial. Estos resultados concuerdan con la hipótesis formulada sobre el nivel de este proceso, por lo que éste sistema de hipótesis es descartada según COBIT; es decir, se han obtenido resultados sobresalientes producto de la forma de gestionar los recursos de la división de sistemas.

4. El 45,45\% del personal encuestado considera que el proceso de definición de procesos, organización y relaciones de TI se encuentra en un nivel definido, mientras que el $26,67 \%$ se encuentra en un nivel administrado y el $20,0 \%$ en un nivel repetible. Estos resultados no concuerdan con la hipótesis formulada sobre el nivel de este proceso, toda vez que la hipótesis postulaba que se encontraría en un nivel inicial, siendo los resultados más que alentadores; esto se debería a que se está avanzando en documentación y socialización de toda la tecnología de información y comunicaciones de la universidad.

5. El 45,45\% del personal encuestado considera que el proceso de definición evaluación y administración de los riesgos de TI se encuentra en un nivel administrado, mientras que el $18,18 \%$ se encuentra en un nivel definido y el $27,27 \%$ en un nivel repetible. Estos resultados no concuerdan con la hipótesis formulada sobre el nivel de este proceso; es decir, la organización está consciente de sus responsabilidades y obligaciones legales y contractuales y considera la importancia de los riesgos de TI, así como las políticas de uso de TIC en la universidad. 


\section{NOTAS}

1 MÁrQueZ P. La TIC y sus aportaciones a la sociedad. [Monografía en Internet]. Barcelona. Universidad Autónoma de Barcelona, Departamento de pedagogía aplicada. 08/23/03. [Citada 05 de Octubre 2011]. [Una página digital]. Disponible desde: http://www.pangea.org/ peremarques/tic.htm

2 IBERMÁtiCA. Evolución de la TIC: Oportunidades y amenazas sociales. [Artículo en Internet]. España. Ibermática. 2006. [Citada 05 de Octubre 2011]. [Una página digital]. Disponible desde: http://www.ibermatica.com/ibermatica/eventos/2006/mtevolucionticsoportunidadesamenazas

3 CoCA J. La TIC crecen en el mundo al 30\% anual. [Artículo en Internet]. España. Telefónica. 2008. [Citada 09 de Octubre 2011]. Disponible desde: http://www.tendencias21.net/Las-TICscrecen-en-el-mundo-al-30-anual_a3164.html

4 Morales R. La UIT promueve un Plan Marshall de Internet para África. [Artículo en Internet]. España. Telefónica. 2008. [Citada Citada 07 de Octubre 2011]. Disponible desde: http://www. tendencias21.net/La-UIT-promueve-un-Plan-Marshall-de-Internet-paraafrica_a1784.html

5 SAlazAR C. La TIC como herramienta a la gestión empresarial. [Artículo en Internet]. Chile. Universidad Austral de Chile, Instituto Académico de Administración. 2008. [Citada 05 de Octubre 2011]. Disponible desde: http://cibermundos.bligoo.com/content/view/145501

6 Medina G. Inversión en TIC en el Perú. [Noticia en Internet]. Lima. Agencia Press Perú. [Citada 15 de Octubre 2011]. Disponible desde: http://www.peruenvideos.com/intel-recomiendo-a-peruinvertir-en-las-tics/

7 InSTITUTO DE MARKETING Y ESTUdios S.L. Conocimiento y utilización de las tecnologías de la información y la comunicación (TIC) en los emprendedores y microempresas apoyadas por el proyecto MICRO [monografía en internet]. España: Instituto de Marketing y Estudios S.L. 2004 [citada 15 octubre 2011]. Disponible desde: www.emicromurcia.com/micro/modules.phpname $=$ Downloads\&d_op $=$ getit\&lid $=62$

8 El TI GOVERNANCE INSTITUTE. Objetivos de control para la información y tecnologías relacionadas (COBIT, en inglés: Control Objectives for Information and Related Technology) [Archivo en Internet]. United States of America: TI Governance Institute. 2007 [Citada 05 de Octubre 2011] Disponible desde: http://cs.uns.edu.ar/ ece/auditoria/cobiT4.1spanish.pdf

9 VARAS P. Perfil de planeamiento y organización de tecnologías de la información y comunicación (TIC) en la empresa Acuapesca SAC de la provincia de Casma. [Tesis para optar el título de ingeniero de sistemas]. Chimbote: Universidad Católica Los Ángeles de Chimbote; [citada 04 Ener. 2013]. 2009.

10 Castro M. Nivel de planeamiento y organización de las tecnologías de información y comunicación (TIC) en el hospital La Caleta de Chimbote año 2011. [Tesis para optar el título de ingeniero de sistemas]. Chimbote: Universidad Católica Los Ángeles de Chimbote [citada 04 Ene. 2013]. 2009.

11 Zegarra. J. Nivel de conocimiento del personal y uso de las tecnologías de información y comunicación (TIC) en la Municipalidad Distrital de Santa en el año 2009. [Tesis para optar el título de Ingeniero de Sistemas]. Chimbote: Universidad Católica Los Ángeles de Chimbote; [Citada 15 Feb. 2013]. 2009.

12 Benites G. Nivel de conocimiento del personal y uso de las tecnologías de información y comunicación (TIC) en la Municipalidad Distrital de Coishco en el año 2009. [Tesis para optar el título de ingeniero de sistemas]. Universidad Católica los Ángeles de Chimbote [citada 06 Mar. 2013]. 2009. 